ENFOQUES JURÍDICOS

REVISTA MULTIDISCIPLINAR DEL CEDEGS

ISSN 26832070

Número 04

julio-diciembre 2021
Reseña: "Segundo Congreso de Derecho Constitucional Iberoamericano. Preludio a Cátedra Mahatma Gandhi-Universidad Veracruzana"

Rita María León López

DOI: https://doi.org/10.25009/ej.v0i04.2576

\title{
Segundo Congreso de Derecho Constitucional Iberoamericano Preludio a Cátedra Mahatma Gandhi-Universidad Veracruzana
}

Recibido 28 junio 2021-Aceptado 29 junio 2021

II Congreso internacional de Derecho constitucional Iberoamericano. (23-24 de febrero de 2021). "Crisis constitucionales. Estados constitucionales de excepción. Cultura de paz". Universidad de Salamanca, Universidad Veracruzana e Instituto Euromexicano. México.

\author{
Rita María León López* \\ Universidad Veracruzana. Xalapa-Veracruz, México \\ rileon@uv.mx
}

Este año de 2021 se llevó a cabo el Congreso en su edición segunda y primera en modalidad virtual por la extraordinaria condición de los últimos tiempos. Para tal finalidad se utilizó la plataforma virtual Zoom, a través de un enlace y código.

Participaron en su organización las siguientes entidades académicas: Universidad de Salamanca y Universidad Veracruzana, Red Internacional Cuerpos Académicos de Estudios Institucionales José Ramón Cossío Díaz, la Cátedra Mahatma Gandhi y el Instituto Euromexicano.

La organización se conformó por una Dirección Académica con las doctoras: Ángela Figueruelo Burrieza y Mercedes Iglesia Bárez de la Universidad de Salamanca, y, los doctores Marisol Luna Leal y Homero Vázquez Ramos de la Universidad Veracruzana.

Y la Coordinación por los profesores: Pablo Ramos Hernández y Sergio Martín Guardado de la Universidad de Salamanca, así como la doctora Alejandra Verónica Zúñiga Ortega de la Universidad Veracruzana.

Las participaciones contaron con un moderador quien les recordó las recomendaciones:

\footnotetext{
* Académica adscrita al Centro de Estudios sobre Derecho, Globalización y Seguridad de la Universidad Veracruzana.

Urimereas wersenasu
}


ENFOQUES JURIDICOS

REVISTA MULTIDISCIPLINAR DEL CEDECS

ISSN 26832070
Reseña: "Segundo Congreso de Derecho Constitucional Iberoamericano. Preludio a Cátedra Mahatma Gandhi-Universidad Veracruzana"

Rita María León López

Número 04

DOI: https://doi.org/10.25009/ej.v0i04.2576

Cada uno de los ponentes debió exponer en 10 minutos la reflexión de su tema, al concluir la participación de los integrantes de la mesa, se estableció de acuerdo a la demanda, un periodo de minutos para responder a las preguntas entregadas vía chat en facebook relacionadas a los temas expuestos.

El congreso se desarrolló en seis mesas de trabajo y tres conferencias magistrales. En las siguientes líneas abordaremos los títulos de cada ponencia y los títulos de las mesas de trabajo, y, rescataremos los comentarios principales de las conferencias magistrales de este segundo Congreso Internacional de Derecho constitucional Iberoamericano.

En su cargo de directora del congreso, la doctora Ángela Figueruelo Burrieza, dirigió las primeras palabras y recordó que, en el mes de marzo del año 2018, se llevó a cabo el Primer Congreso Internacional "Retos del constitucionalismo Iberoamericano", año del 40 aniversario de la Constitución española y la celebración de los ochocientos años de la fundación de la Universidad de Salamanca. Al clausurar ese primer congreso, se propusieron realizarlo cada dos años y en cada una de las universidades participantes. La Universidad Veracruzana se postuló para llevar a cabo la organización del segundo congreso del año 2020, mismo que se postergó por las condiciones presentadas en ese año, realizándolo hasta el presente año de 2021.

“(...) hoy con el mismo éxito que entonces (...), podemos comenzar este Segundo Congreso Internacional de Derecho Constitucional Iberoamericano. En el primero abordamos con carácter general los retos del constitucionalismo. En esta ocasión abordaremos auténticos retos que nos han dificultado la convivencia y nos van hacer más difíciles el día a día de cara al futuro, se va hablar de crisis constitucionales, de estados constitucionales, de excepción, de cultura de paz.

Les auguró que la intervención de todas y todos sea exitosa y que el público que nos escucha quede tremendamente satisfecho y podamos en un par de años volver a reunirnos ya presencialmente. La fecha que hoy hemos elegido para comenzar este congreso tiene un doble sentido en nuestro país, hace unos años en el 81, el 23 de febrero concretamente hace 40 años, estuvimos a punto de perder la recién estrenada democracia con el golpe de estado que se pronunció en el Congreso de los Diputados a las cinco y pico de la tarde, salimos de aquel bache y hemos vivido otros 40 años en paz, hagamos votos como ciudadanas y ciudadanos de democracias consolidadas para que podamos seguir viviendo en paz, para que solventemos las crisis constitucionales para que no tengamos que seguir en estados excepcionales y podamos vivir en culturas de paz.

Enseguida correspondió el turno de las doctoras Mercedes Iglesias Bárez y Marisol Luna Leal, y agradecieron el mutuo apoyo para la organización de este evento. Se resalta del

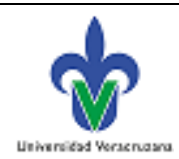

pp. $142-148$

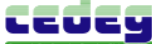


ENFOQUES JURIDICOS

REVISTA MULTIDISCIPLINAR DEL CEDECS

ISSN 26832070
Reseña: "Segundo Congreso de Derecho Constitucional Iberoamericano. Preludio a Cátedra Mahatma Gandhi-Universidad Veracruzana"

Rita María León López

DOI: https://doi.org/10.25009/ej.v0i04.2576

discurso de la Dra. Luna, lo concerniente a la Cátedra Mahatma Gandhi y su primera participación en un evento académico.

\section{Conferencia magistral. Dra. Ángela Figueruelo Burrieza}

"La defensa política de la constitución en tiempos de pandemia"

Moderadora: Mercedes Iglesias Bárez

De esta conferencia se extrae lo siguiente, "la pandemia mundial (...), el Estado español se encuentra en esa situación y [utiliza] la legislación extraordinaria, nuestro gobierno proclama el estado de alarma el día 14 de marzo (...).

Los controles de estas medidas en las cuales se han visto restringidos, modulados, suspendidos, etc., muchos derechos fundamentales, plantean dudas desde el punto de vista del derecho constitucional. La doctrina se divide, nadie esta conforme al cien por cien de la declaración del estado de alarma".

Explica que el estado de alarma "tiene como efecto, como consecuencia limitar los derechos fundamentales, aquellos que figuran en la declaración a los efectos de solventar la situación excepcional por la cual atravesamos. El estado de excepción, en cambio, permite suspender algunos de los derechos que estén previstos en esa declaración.

Ahora bien, si el estado de alarma permite limitar derechos y, se puede apoyar en causas producidas por fenómenos naturales, como puede ser una pandemia y una crisis sanitaria en el primer periodo de la declaración del estado de alarma con el confinamiento total de la población. Hay que preguntarse y analizar muy seriamente, qué entendemos por suspender y qué entendemos por limitar, porque aquí hay apreciaciones subjetivas de valoración de esos términos. Si hay un confinamiento total en sus domicilios de la población es una limitación de la libertad de movimiento o es una suspensión del derecho a la movilidad, el derecho al tránsito".

En la parte final de su discurso, se pregunta ¿hemos podido afrontar de forma correcta la situación de pandemia mundial por la que atravesamos? y expresa sus dudas, y agrega que, "debemos estar preparados para hacer frente a otros problemas que se nos plantean, y sinceramente las democracias consolidadas del occidente, en mi opinión, no tienen las suficientes herramientas jurídicas para hacer frente a esta pandemia, hay que pensar en estos momentos, sin prisas pero sin pausas, cómo debemos de responder a esos instrumentos, ósea qué instrumentos jurídicos debemos crear, tanto a nivel nacional como a nivel internacional (...), no pensar únicamente a nivel interno, sino también a nivel internacional. (...) lo que sí me temo es que, muchos de los derechos que se nos han visto limitados y que se nos han visto cercenados tardemos bastante tiempo en volver a conseguir el nivel de ellos que teníamos, yo hago votos para que las libertades no peligren, para que la dignidad de la persona que es fundamento del orden político y de la paz social,

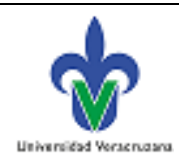

pp. $142-148$ 
ENFOQUES JURIDICOS REVISTA MULTIDISCIPLINAR DEL CEDECS

ISSN 26832070

Número 04

julio-diciembre 2021
Reseña: "Segundo Congreso de Derecho Constitucional Iberoamericano. Preludio a Cátedra Mahatma Gandhi-Universidad Veracruzana"

\section{Rita María León López}

DOI: https://doi.org/10.25009/ej.v0i04.2576

siga estando reconocido en nuestros representantes políticos". Y finalizó su participación agradeciendo e inaugurando el congreso.

\section{Tabla de la primera y segunda mesa de trabajo}

\begin{tabular}{|c|c|}
\hline \multicolumn{2}{|r|}{23 FEBRERO 2021} \\
\hline \multicolumn{2}{|c|}{$\begin{array}{l}\text { MESA 1: DERECHO INTERNACIONAL, CONVENCIONAL Y CONSTITUCIONAL DE EXCEPCIÓN } \\
\text { Moderador: Pablo Ramos. Universidad de Salamanca }\end{array}$} \\
\hline PARTICIPANTE & PONENCIA \\
\hline $\begin{array}{l}\text { - Mercedes Iglesias Bárez-Universidad de } \\
\text { Salamanca-España }\end{array}$ & $\begin{array}{l}\text { Democracia, Elecciones y Pandemia en el Consejo de Europa y la Comisión de } \\
\text { Venecia }\end{array}$ \\
\hline $\begin{array}{l}\text { - Augusto Marcelo Santis Poblete-Universidad } \\
\text { Autónoma de Chile-Chile }\end{array}$ & $\begin{array}{l}\text { Nueva Constitución para Chile en tiempos de estallido social y pandemia: } \\
\text { Escenario actual y proyecciones }\end{array}$ \\
\hline $\begin{array}{l}\text { - Juan Manuel Bautista Jiménez-Universidad de } \\
\text { Salamanca-España }\end{array}$ & $\begin{array}{l}\text { Limitaciones a los derechos humanos durante la pandemia en España y } \\
\text { Derecho internacional }\end{array}$ \\
\hline $\begin{array}{l}\text { - Enrique Serrano Salazar-Universidad La Salle de } \\
\text { Pachuca-México }\end{array}$ & El nombre "Democracia", una interpretación constitucional \\
\hline $\begin{array}{l}\text { - Juan Daniel Elorza Saravia-Universidad de } \\
\text { Salamanca-España }\end{array}$ & Viralidad virtual, polarización real y Estado de Derecho \\
\hline $\begin{array}{l}\text { - Agassiz Almeida Filho-Universidade Estadual da } \\
\text { Paraíba-UEPB-Brasil }\end{array}$ & Estado deexcepción en red y lavajatismo \\
\hline $\begin{array}{l}\text { - Jorge Benavides Ordóñez-Instituto de Altos } \\
\text { Estudios Nacionales-IAEN-Ecuador }\end{array}$ & Control constitucional y estados de excepción en Ecuador por el COVID-19 \\
\hline $\begin{array}{l}\text { - Sergio Arnoldo Morán Navarro-Universidad } \\
\text { Autónoma de Nayarit-México }\end{array}$ & $\begin{array}{l}\text { Los límites al derecho a la libertad personal, la inconvencionalidad de la figura } \\
\text { del arraigo en México }\end{array}$ \\
\hline $\begin{array}{l}\text { - Luis Andrés Cucarella Galiana-Universidad de } \\
\text { Valencia-España }\end{array}$ & Paz, Derecho Procesal y oportunidad criminal \\
\hline \multicolumn{2}{|c|}{$\begin{array}{c}\text { MESAS 2: CRISIS CONSTITUCIONALES, DERECHO DE EXCEPCIÓN Y DIVISIÓN DE PODERES HORIZONTAL } \\
\text { Moderadora: Rebeca Elizabeth Contreras López-Universidad Veracruzana }\end{array}$} \\
\hline $\begin{array}{l}\text { - Michael Gustavo Núñez Torres-Universidad } \\
\text { Autónoma de Nuevo León-México }\end{array}$ & $\begin{array}{l}\text { La capacidad legislativa del Gobierno en situaciones de excepción y las } \\
\text { garantías a los derechos humanos: El caso del Covid en México }\end{array}$ \\
\hline $\begin{array}{l}\text { - Aldo Rafael Medina García-Universidad } \\
\text { Autónoma de Nayarit-México } \\
\end{array}$ & División y control del poder en la excepción constitucional \\
\hline $\begin{array}{l}\text { - Ana Lilia Ulloa Cuéllar-Instituto de } \\
\text { Investigaciones Jurídicas-México }\end{array}$ & $\begin{array}{l}\text { La recuperación de la integridad en el constitucionalismo contemporáneo. } \\
\text { Construyendo un constitucionalismo "D" }\end{array}$ \\
\hline $\begin{array}{l}\text { - Pamela Lilí Fernández Reyes-Universidad } \\
\text { Autónoma de Nayarit-México }\end{array}$ & $\begin{array}{l}\text { La división de poderes en el constitucionalismo mexicano ¿simulación o } \\
\text { realidad en la política mexicana? }\end{array}$ \\
\hline $\begin{array}{l}\text { - Miguel Ángel Rodríguez Vázquez-Universidad } \\
\text { Juárez del Estado de Durango-México }\end{array}$ & $\begin{array}{l}\text { Uso de facultades extraordinarias en México. Análisis desde una perspectiva } \\
\text { histórico-constitucional }\end{array}$ \\
\hline $\begin{array}{l}\text { - Homero Vázquez Ramos-Universidad de } \\
\text { Guadalajara-México }\end{array}$ & ¿Están dadas las condiciones en México para una nueva constitución política? \\
\hline $\begin{array}{l}\text { - Enrique Córdoba del Valle-Facultad de Derecho. } \\
\text { Universidad Veracruzana-México }\end{array}$ & ¿Reconstrucción o división de poderes? \\
\hline $\begin{array}{l}\text { - David Quintano Díaz-El Colegio de Veracruz- } \\
\text { México }\end{array}$ & Derecho de excepción en el marco del Derecho Administrativo \\
\hline
\end{tabular}

La segunda Conferencia magistral a cargo del Dr. José Ramón Cossío Díaz, se encuentra publicada en el presente número.
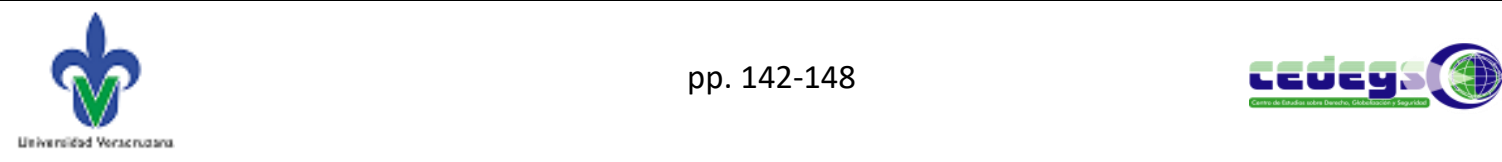
ENFOQUES JURIDICOS REVISTA MULTIDISCIPLINAR DEL CEDECS

ISSN 26832070

Número 04

julio-diciembre 2021
Reseña: "Segundo Congreso de Derecho Constitucional Iberoamericano. Preludio a Cátedra Mahatma Gandhi-Universidad Veracruzana"

Rita María León López

DOI: https://doi.org/10.25009/ej.v0i04.2576

2. Tabla de la tercera y cuarta mesa de trabajo

\begin{tabular}{|c|c|}
\hline \multicolumn{2}{|r|}{24 FEBRERO 2021} \\
\hline \multicolumn{2}{|c|}{$\begin{array}{l}\text { MESAS 3: CRISIS CONSTITUCIONALES, DERECHO DE EXCEPCIÓN Y DISTORSIONES EN LA DISTRIBUCIÓN TERRITORIAL DEL PODER } \\
\text { Moderador: Sergio Martín Guardado-Universidad Salamanca }\end{array}$} \\
\hline PARTICIPANTES & PONENCIAS \\
\hline $\begin{array}{l}\text { - Marisol Luna Leal-Universidad Veracruzana- } \\
\text { México }\end{array}$ & Federalismo mexicano: Entre la desarticulación y la irrupción \\
\hline $\begin{array}{l}\text { - Pedro Rubén Torres Estrada-Escuela de Gobierno } \\
\text { y Trasformación Pública del TEC de Monterrey- } \\
\text { México }\end{array}$ & Las tensiones que ha generado el Covid-19 en el modelo federal Mexicano \\
\hline $\begin{array}{l}\text { - Arturo Centeno Valencia-Escuela de Gobierno y } \\
\text { Transformación Pública del Tec de Monterrey- } \\
\text { México }\end{array}$ & $\begin{array}{l}\text { ¿Son constitucionales las restricciones a los derechos humanos por autoridades } \\
\text { locales? }\end{array}$ \\
\hline \multicolumn{2}{|c|}{ MESAS 4: DERECHO DE EXCEPCIÓN Y SUSPENSIÓN DE DERECHOS FUNDAMENTALES Y DERECHOS HUMANOS (I) } \\
\hline $\begin{array}{l}\text { - Marina del Pilar Olmeda García-Universidad } \\
\text { Autónoma de Baja California-México }\end{array}$ & Los retos de los Derechos Humanos frente al Covid-19 \\
\hline $\begin{array}{l}\text { - Irina Graciela Cervantes Bravo-Universidad de } \\
\text { Nayarit-México }\end{array}$ & Elecciones y Derechos Intrapartidistas en tiempos de pandemia en México \\
\hline $\begin{array}{l}\text { - Graciela Guadalupe Buchanan Ortega-Poder } \\
\text { Judicial del Estado de Nuevo León-México }\end{array}$ & Efectos de la Pandemia en la Violencia Doméstica \\
\hline $\begin{array}{l}\text { - Lilián Galván Bautista-Universidad de Salamanca- } \\
\text { España }\end{array}$ & Las garantías de los derechos fundamentales en el nuevo paradigma \\
\hline $\begin{array}{l}\text { - Gonzalo Armienta Hernández-Universidad } \\
\text { Autónoma de Sinaloa-México }\end{array}$ & $\begin{array}{l}\text { La ponderación de los derechos humanos y el derecho de excepción y } \\
\text { suspensión }\end{array}$ \\
\hline $\begin{array}{l}\text { - Danielle da Rocha Cruz-Universidade Federal da } \\
\text { Paraíba-Brasil }\end{array}$ & $\begin{array}{l}\text { Delitos de mera desobediencia, leyes penales en blanco y derecho de } \\
\text { excepción en tiempos de pandemia }\end{array}$ \\
\hline $\begin{array}{l}\text { - Julia Sevilla Merino-Universidad de Valencia- } \\
\text { España }\end{array}$ & Perspectiva de género en la suspensión de derechos en tiempos de pandemia \\
\hline $\begin{array}{l}\text { - Ricardo Maurício Freire Soares-Universidade } \\
\text { Federal da Bahia-Brasil } \\
\end{array}$ & $\begin{array}{l}\text { Direitos Fundamentais e Crise Sanitária no Brasil: a necessidade de um Estado } \\
\text { Solidarista }\end{array}$ \\
\hline $\begin{array}{l}\text { - Marcelo Eugênio Feitosa Almeida-Universidade } 7 \\
\text { de Setembro-Brasil }\end{array}$ & $\begin{array}{l}\text { Pandemia, violación masiva de derechos fundamentales y responsabilidad } \\
\text { política según el régimen jurídico-constitucional brasileño }\end{array}$ \\
\hline - Sergio Alexandre Camargo-Brasil & Redimensión de los derechos fundamentales en las democracias actuales \\
\hline $\begin{array}{l}\text { - Marta León Alonso-Universidad de Salamanca- } \\
\text { España }\end{array}$ & Conflictos de derechos en situaciones de emergencia sanitaria \\
\hline $\begin{array}{l}\text { - Julio César Arango Chontal-Universidad } \\
\text { Veracruzana-México }\end{array}$ & Supuestos de la restricción o suspensión del derecho a la educación en México \\
\hline $\begin{array}{l}\text { - Pablo Latorre Rodríguez / Karen Yarely García } \\
\text { Arizaga-Universidad Autónoma de Baja } \\
\text { California-México }\end{array}$ & $\begin{array}{l}\text { El Estado de Excepción y la limitación de derechos. Breve aproximación a las } \\
\text { regulaciones española y mexicana }\end{array}$ \\
\hline $\begin{array}{l}\text { - Judith Aguirre Moreno / Manilo Fabio Casarín } \\
\text { León-Universidad Veracruzana-México }\end{array}$ & Constitucionalismo y pandemia: tensiones y desafíos en el Estado democrático \\
\hline
\end{tabular}

\section{Conferencia Magistral: Dra. Tica Font - 25 de febrero 2021}

Asociación Española De Investigadores Por La Paz (Aipaz)-España

Moderadora: Marisol Luna Leal

Planteó su exposición en tres bloques: el concepto de paz, el concepto de no violencia y los retos en el ámbito jurídico con la no violencia, no tanto con la paz.

El concepto de paz en la antigua Grecia, la llamaban Irene, y la sociedad griega concebía la paz como un estado de tranquilidad, de serenidad, de ausencia de perturbaciones, pero

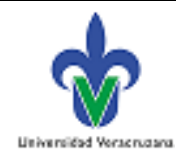


ENFOQUES JURIDICOS

REVISTA MULTIDISCIPLINAR DEL CEDECS

ISSN 26832070
Reseña: "Segundo Congreso de Derecho Constitucional Iberoamericano. Preludio a Cátedra Mahatma Gandhi-Universidad Veracruzana"

Rita María León López

Número 04

DOI: https://doi.org/10.25009/ej.v0i04.2576

de alguna manera fueron construyendo la paz como ausencia de hostilidades, de ausencia de guerra entre ciudades, de alguna manera se encuentran dos vertientes: una especie de armonía interior y una especie de armonía social. Los griegos se mantenían en paz con los miembros de su sociedad, no disfrutaban de ese estado los no miembros de la sociedad griega.

En la sociedad romana, la paz se encontraba y mantenía en el interior del imperio, mientras en su entorno tenían ausencia de paz, o mantenían una paz armada.

Las sociedades tenían un marcado concepto jurídico, buscaban la ausencia de rebeliones, también como un equivalente de seguridad, si hay paz hay seguridad.

Con la finalización de la guerra fría, acuño otro termino a la paz, u otro adjetivo a la paz, la de liberar o vencedor de la guerra fría, estos vencedores podrían imponer tres elementos: la democracia, sistema económico capitalista y el libre mercado.

Señaló que el sociólogo noruego Johan Galtung, designo el triángulo de la violencia, la paz negativa esta definida como la ausencia de guerra, lo contrario de la paz es la violencia. [esto es:] La violencia directa, la violencia estructural (sociedad) y la violencia cultural.

La paz social es un concepto dinámico, que siempre esta en construcción, y se realizan acciones transformadoras.

No violencia es un método de acción sociopolítica, método de transformación social que se pueden utilizar actos de omisión o actos de comisión o combinación de actos. Se divide en dos bloques: la no violencia ética o la no violencia holística y la no violencia pragmática; ambas violencias apuestan por superar los conflictos, persiguen una sociedad justa e igualitaria, a través de métodos pacíficos, y rechazan el uso de la violencia física para combatir una injusticia. La utilización de técnicas como huelgas, manifestaciones, entre otras como instrumentos, a garantizar la lucha de trabajadores, ganar derechos civiles, etc.

De alguna manera la violencia civil como derecho, y sería un acto público, no violento, consciente, contrario a la ley, cometido con el propósito de un cambio de ley o en los programas de gobierno. Por qué un acto público, porque no sería un acto clandestino, sería con responsabilidad que asumes desobedeciendo. Todas las acciones no violentas, la gente se tiene que preparar, yo veo que nuestra cultura es la de negarlo todo; nadie ha hecho nada, los políticos tampoco, las personas tampoco, yo creo que tiene tanto la desobediencia.

(...) analizado el régimen jurídico, yo creo que sí, que se están sentando las bases de que cuando haya acciones de carácter no violento, hacía el poder político son más punibles, que cuando estas acciones se hacen en la esfera económica, en la esfera económica estas acciones hacia el mundo empresarial o de la economía, no están tan penalizadas que cuando se llevan a cabo acciones hacia diríamos el ámbito político".

Del anterior descernimiento argumento que "eso lo deben decir vosotros, si lo que yo digo es cierto o, es una percepción errónea o no". De esta manera concluyo su charla.

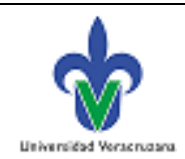

pp. $142-148$ 
ENFOQUES JURIDICOS REVISTA MULTIDISCIPLINAR DEL CEDECS

ISSN 26832070

Número 04

julio-diciembre 2021

\section{Reseña: "Segundo Congreso de Derecho Constitucional Iberoamericano. Preludio a Cátedra Mahatma Gandhi-Universidad Veracruzana"}

\section{Rita María León López}

DOI: https://doi.org/10.25009/ej.v0i04.2576

\section{Tabla de la quinta y sexta mesa de trabajo}

\begin{tabular}{|c|c|}
\hline \multicolumn{2}{|r|}{25 FEBRERO 2021} \\
\hline \multicolumn{2}{|c|}{$\begin{array}{c}\text { MESAS 5: DERECHO DE EXCEPCIÓN Y SUSPENSIÓN DE DERECHOS FUNDAMENTALES Y DERECHOS HUMANOS (II) } \\
\text { Moderadora: Laura Hernández-Universidad Salamanca }\end{array}$} \\
\hline PARTICIPANTES & PONENCIAS \\
\hline $\begin{array}{l}\text { - Rocío González Pereyra-Benemérita Universidad } \\
\text { Autónoma de Puebla-México }\end{array}$ & Derecho, mujeres y trabajo en México en tiempos de pandemia \\
\hline $\begin{array}{l}\text { - Melissa Gómez Díaz-Instituto de Investigaciones } \\
\text { Jurídicas-México }\end{array}$ & $\begin{array}{l}\text { El concepto de superación general de los derechos fundamentales en el estado } \\
\text { de excepción }\end{array}$ \\
\hline $\begin{array}{l}\text { - José Alfredo Gómez Reyes-Universidad de } \\
\text { Quintana Roo-México }\end{array}$ & La Justicia en Línea como Derecho Humano de acceso a la Justicia \\
\hline $\begin{array}{l}\text { - Karla Elizabeth Mariscal Ureta-Universidad } \\
\text { Autónoma de Querétaro-México }\end{array}$ & $\begin{array}{l}\text { Derechos humanos y ciudadanía sostenible en tiempos de Pandemia, una } \\
\text { aproximación mexicana }\end{array}$ \\
\hline \multicolumn{2}{|c|}{$\begin{array}{l}\text { MESAS 6: CULTURA DE PAZ Y RESISTENCIAS NO VIOLENTAS. NOVIOLENCIA. NO-VIOLENCIA } \\
\text { Moderadoras: Laura Hernández-Universidad Salamanca / Alejandra V. Zúñiga Ortega-Universidad Veracruzana }\end{array}$} \\
\hline $\begin{array}{l}\text { - Alejandra V. Zúñiga Ortega-Universidad } \\
\text { Veracruzana-México }\end{array}$ & La construcción de una Cultura de Paz en México \\
\hline $\begin{array}{l}\text { - Ivette Mecott Rivera-Universidad Autónoma de } \\
\text { Nuevo León-México }\end{array}$ & $\begin{array}{l}\text { El heteropatriarcado como factor disruptor en la cultura de la paz. Una } \\
\text { aproximación desde las nuevas masculinidades }\end{array}$ \\
\hline $\begin{array}{l}\text { - Julio Cabrera Dircio / María Ivonne Hernández } \\
\text { Muñoz-Universidad Autónoma del Estado de } \\
\text { Morelos-México }\end{array}$ & La mediación un recurso constitucional para construir la Cultura de Paz \\
\hline $\begin{array}{l}\text { - Rebeca Contreras López-CEDEGS. Universidad } \\
\text { Veracruzana-México }\end{array}$ & La justicia restaurativa, una vía para promover la cultura de la paz \\
\hline $\begin{array}{l}\text { - Carlos Alberto Prieto Godoy / Juan Miguel } \\
\text { Salcedo Rosales-Universidad Autónoma de } \\
\text { Nayarit-México }\end{array}$ & $\begin{array}{l}\text { La función mediadora del ACNUR en la justicia administrativa y los derechos } \\
\text { humanos }\end{array}$ \\
\hline $\begin{array}{l}\text { - Lorena Zaleta Morales-Universidad Autónoma } \\
\text { del Carmen-México }\end{array}$ & La formación de una cultura para la paz en la educación \\
\hline $\begin{array}{l}\text { - Juan Pablo Luna Leal-Universidad Humanitas. } \\
\text { Campus Querétaro-México }\end{array}$ & La mediación escolar ante la pandemia generada por el virus SARS-COV-2 \\
\hline $\begin{array}{l}\text { - Laura Patricia Hernández Vigueras-Instituto de } \\
\text { Mediación Veracruzano-México }\end{array}$ & Reflexiones sobre Mediación en la principal Institución Socializadora: la familia \\
\hline $\begin{array}{l}\text { - Guillermo Sánchez Vaca-Facultad de Derecho. } \\
\text { Universidad Veracruzana-México }\end{array}$ & Marginación y cultura de paz \\
\hline $\begin{array}{l}\text { - Austria Paola Barradas-Facultad de Derecho. } \\
\text { Universidad Veracruzana-México }\end{array}$ & $\begin{array}{l}\text { Mediación penal en línea y la justicia restaurativa: perspectivas y retos en } \\
\text { México }\end{array}$ \\
\hline $\begin{array}{l}\text { - María Elena Reyes Monjarás / Daniel Antonio } \\
\text { González Hernández-Universidad Autónoma del } \\
\text { Carmen-México }\end{array}$ & La Cultura de Paz: Desafío para México \\
\hline $\begin{array}{l}\text { - Adriana Segura Casados-Universidad del Golfo } \\
\text { de México-México }\end{array}$ & La justicia social y la resiliencia como mecanismos para La Paz \\
\hline $\begin{array}{l}\text { - Ysela Rejon Jiménez / Francisco Javier Tejero } \\
\text { Bolón-Universidad Autónoma del Carmen- } \\
\text { México }\end{array}$ & Una cultura para la paz desde la educación \\
\hline
\end{tabular}

